SHORT REPORT

\title{
Decision making in humans: the effect of manipulating the central noradrenergic system
}

\author{
R E O'Carroll, B P Papps
}

J Neurol Neurosurg Psychiatry 2003;74:376-378

Objectives: Damasio has proposed an influential model of human decision making - the Somatic Marker Hypothesis (SMH), where he argues that somatic feedback to the brain influences decision making in humans. It is proposed that when choosing between options that differ in relative risk, a somatic marker (for example, a "gut feeling") feeds back to the brain and influences cognitive appraisal. This study tested the hypothesis that the noradrenergic system may play a part in this afferent feedback process.

Methods: Thirty healthy young adults were randomly allocated to receive placebo, $4 \mathrm{mg}$, or $8 \mathrm{mg}$ reboxetine /a new selective noradrenergic reuptake inhibitor that acts to increase central noradrenergic activity). It is hypothesised that enhancement of central noradrenergic activity may amplify the effect of afferent feedback, and lead to altered decision making. All subjects completed the lowa Gambling Task (IGT), a computerised card playing game where the player is instructed to try and win as much money as possible over 100 selections from one of four decks.

Results: There were no significant differences between the three groups in gambling task performance.

Conclusion: This result suggests that central noradrenergic activity may not be critically involved in the afferent feedback mechanism that is central to the somatic marker hypothesis.

O ne of the most influential models in contemporary cognitive neuroscience is the somatic marker hypothesis ( $\mathrm{SMH})$, developed by Damasio and colleagues, who argue that somatic feedback to the brain influences higher cognitive processes, in particular, decision making in humans. ${ }^{1-3}$ Damasio proposes that when choosing between options that differ in relative risk, a somatic marker (for example, a "gut feeling") feeds back to the brain and influences cognitive appraisal, often without conscious awareness.

There has been an increasing focus on the role of physiological feedback influencing conscious and unconscious experience in humans. Patients who have suffered total spinal cord transection have drastically reduced feedback from periphery to brain and for many years the effects of spinal transection on psychological functioning have been debated, but there has been comparatively little human experimental work in this area.

As spinal injury patients have reduced somatic/peripheral feedback via the spinal cord, we predicted, based on the SMH, that they may demonstrate riskier behaviour than controls. Damasio stated, "Were you to cut only the signals from the body proper to the brain, your mind would change too. Even partial blocking of brain-body traffic, as happens in patients with spinal cord injury, causes changes in mind state" ${ }^{1}$ We therefore conducted a study ${ }^{4}$ where 20 patients with complete
C6 level spinal cord transection were compared with 20 age, sex, and educationally matched control subjects. The SMH was tested using the Iowa Gambling Task (IGT), a computerised card playing game where the player is instructed to try and win as much money as possible over 100 selections from one of four decks (A-D). The rules are not disclosed in advance, and the player gradually "learns" that two of the decks are "high risk" (A and B) and lead to significant financial losses. Healthy people learn to avoid the risky decks, whereas patients with medial frontal lobe damage $e^{5-7}$ and those with peripheral neuropathy ${ }^{8}$ select an excessive number from the risky decks and consequently lose money. We found no differences in IGT performance when spinal cord transection patients were compared with matched controls. Similarly, in terms of overall financial outcome, there was no significant between group difference. ${ }^{4}$ This result suggested that Damasio's proposed "somatic marker" may not be transmitted to the brain from the periphery via the spinal cord. Afferent feedback via the cranial nerves and the hormonal route may be equally or more influential than afferent feedback transmitted via the spinal cord.

If "gut feelings" during anticipated risk influence higher cognitive functioning, one possible route is via the noradrenergic system. In the present experiment we elected to manipulate the noradrenergic system as the sympathetic system is activated during the arousal that occurs during risk evaluation. Bechara et al, ${ }^{7}$ have shown that healthy people produce a galvanic skin response when pondering a risky selection from the IGT, before conscious awareness of the relative risk of that particular option. In addition, recent work has shown that during periods of emotional arousal, noradrenaline is released within the amygdala. ${ }^{9}$ This may function to "mark" the experience as important and memorable-central noradrenergic release may thus play a part in modulating the encoding of such memories. ${ }^{10}$

The SMH proposes that neurotransmitter systems influence the operation of a neural system for decision making in humans. Recently, Bechara et al ${ }^{11}$ manipulated the serotonin and dopamine system and investigated the effects on gambling task performance. They reported that blockade of both dopamine and serotonin interfered with selection of advantageous choices. However, stimulation of both dopamine and serotonin improved the selection of advantageous choices. Dopamine stimulation improved performance only on the early part of the task, when decisions are thought to be guided by covert knowledge. In contrast, serotonin stimulation improved performance only on the latter part of the task, when decisions are guided by conscious knowledge of which choices are "good" or "bad". ${ }^{11}$ In light of these findings, we tested the hypothesis that the noradrenergic system may also be part of the neurobiological process underlying decision making in humans.

Abbreviations: $\mathrm{SMH}$, somatic marker hypothesis; IGT, lowa Gambling task 
Table 1 Comparison of the three subject groups on potential confounding variables. Mean (SD)

\begin{tabular}{llllll}
\hline & $8 \mathrm{mg}$ Reboxetine & 4 mg Reboxetine & Placebo & $F$ & $\mathrm{p}$ \\
\hline Age & $21.8(4.6)$ & $19.3(0.9)$ & $20.0(3.1)$ & 1.48 & 0.246 \\
Sex & $2 \mathrm{~F}, 9 \mathrm{M}$ & $2 \mathrm{~F}, 6 \mathrm{M}$ & $5 \mathrm{~F}, 6 \mathrm{M}$ & $\chi^{2} 2.07$ & 0.36 \\
$\mathrm{~N}$ & $11.5(4.7)$ & $11.3(4.8)$ & $10.9(4.5)$ & 0.052 & 0.95 \\
$\mathrm{E}$ & $12.9(5.1)$ & $13.4(6.4)$ & $16.6(2.1)$ & 1.99 & 0.16 \\
$\mathrm{P}$ & $5.4(3.4)$ & $5.9(3.8)$ & $4.6(3.4)$ & 0.30 & 0.74 \\
$\mathrm{~L}$ & $3.6(2.2)$ & $4.5(5.9)$ & $2.7(1.9)$ & 0.62 & 0.55 \\
NART IQ & $107.4(6.2)$ & $109.4(5.1)$ & $107.2(3.1)$ & 1.48 & 0.25 \\
\hline
\end{tabular}

N, Eysenck Personality Questionnaire (EPQ) Neuroticism score; E, EPQ Extroversion score; $P, E P Q$ Psychoticism score; L, EPQ Lie score; NART, National Adult Reading Test estimated verbal intelligence quotient.

\section{HYPOTHESIS}

Manipulation of the central noradrenergic system will influence IGT performance. Specifically, noradrenergic reuptake inhibition with reboxetine will act to amplify the somatic marker in a dose dependent manner relative to placebo and result in improved task performance. We also predicted that noradrenergic stimulation will lead to improved performance, particularly over the first 20 trials as the rules are gradually learned, relative to placebo.

\section{METHOD \\ Subjects}

As part of a parallel study ${ }^{12} 36$ healthy young adults were recruited from an undergraduate population. Subjects underwent a physical examination and were randomly allocated to one of three groups, to receive placebo, $4 \mathrm{mg}$ reboxetine, or 8 $\mathrm{mg}$ reboxetine. These doses were selected as $8 \mathrm{mg}$ reboxetine is the maximum recommended daily dose for adults suffering from depressive illness, and we wished to test for dose dependent effects. Because of drop out and equipment failure, 30 subjects completed the study, 11 in the $8 \mathrm{mg}$ reboxetine group, 11 in the placebo group, and eight in the $4 \mathrm{mg}$ reboxetine group.

\section{Reboxetine}

Reboxetine is a selective inhibitor of noradrenaline reuptake. This reuptake inhibition results in the increase of noradrenaline availability in the synaptic cleft and enhancement of central noradrenergic transmission. Reboxetine has no significant affinity for adrenergic $\alpha-1, \alpha-2, \beta$, or muscarinic receptors, antagonism of which is associated with cardiovascular, anticholinergic and sedative effects of other antidepressants. Furthermore, no cognitive or psychomotor impairments have been observed with reboxetine in clinical studies, ${ }^{13}$ or in studies with healthy adults ${ }^{14}$ making it a good candidate for use in the assessment the role of the noradrenergic system in higher cognitive functioning.

\section{Procedure}

On arrival at the laboratory, subjects were given a single capsule containing $4 \mathrm{mg}$ reboxetine, $8 \mathrm{mg}$ reboxetine, or placebo. The pharmacokinetics of reboxetine are such that peak serum concentrations occur two hours after drug administration. ${ }^{15}$ Accordingly, all subjects received their capsules two hours before completing the IGT. To confirm random allocation to the three groups, immediately after drug administration and before the drug could plausibly affect test performance, subjects completed the National Adult Reading Test (NART) ${ }^{16}$ to estimate intelligence, and the Eysenck Personality Questionnaire-Revised (EPQ) ${ }^{17}$ to assess personality characteristics.

\section{lowa gambling task}

The IGT was used to test the SMH. It consists of a computerised card playing game where the player is instructed to try

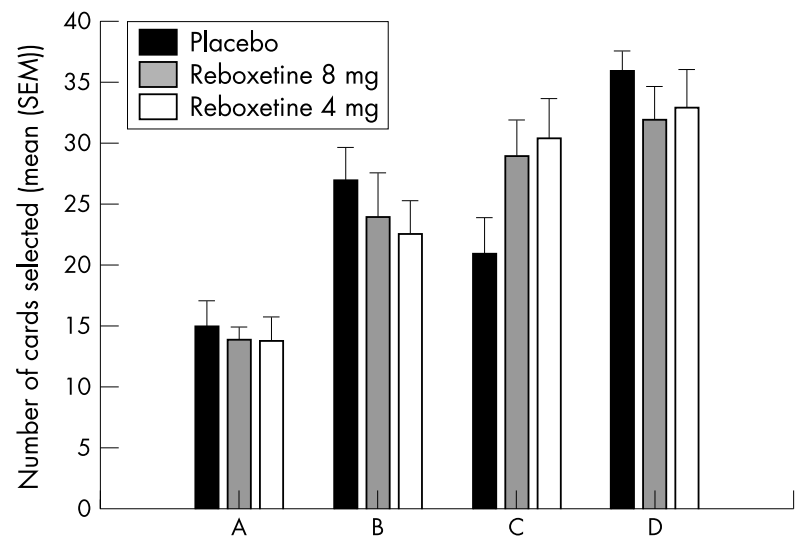

Figure 1 Number of cards selected from each deck by each group. Decks $A$ and $B$ are disadvantageous, $C$ and D are advantageous.

and win as much money as possible over 100 selections from one of four decks. The rules are not disclosed, and the player gradually "learns" that two of the decks are "high risk" (A and B) - that is, intermittently produce large rewards but in the long term lead to significant financial losses, whereas two decks ( $C$ and $D$ ) lead to modest but consistent gains-see Bechara et $a l,{ }^{5}$ for a full description of the task.

\section{RESULTS}

There were no differences between the groups on age, sex ratio, intelligence level, and personality, confirming that the random allocation resulted in three groups that were well matched on potential confounding variables, (see table 1). All subjects in this study completed the IGT at the time of peak drug plasma concentration. The summary of card selection over 100 trials is shown in figure 1 . Repeated measures multivariate analysis of variance (Wilks' $\lambda$ ) revealed that there was no effect of group $F$ $(2,27)=0.00, p=1.00$, a clear effect of card deck $F(3,25)=36.51$, $\mathrm{p}<0.001$, and critically, no group by deck interaction $F$ $(6,50)=1.07, p=0.40$. Similarly, in terms of overall financial outcome, there was no significant difference between the groups. In an attempt to test for between group differences in rate of learning across the five blocks of 20 trials, a strategy score was calculated for each subject for each block by subtracting the number of selections from decks $\mathrm{C}$ and $\mathrm{D}$ (advantageous) from decks A and B (disadvantageous). A repeated measures multivariate analysis of variance was then run on this strategy score across the five blocks. This revealed a clear effect of block, $F(4,24)=15.18, \mathrm{p}<0.001$, but critically no group by block interaction $F(8,48)=0.62, \mathrm{p}=0.761)$ and no effect of group $F$ $(2,27)=0.76, p=0.48$. All three groups gradually learned to avoid the "high risk" decks at an equivalent rate. 


\section{DISCUSSION}

We have tested the hypothesis that enhanced central noradrenergic activity may amplify the effects of afferent feedback/autonomic arousal during risk evaluation in a decision making paradigm. Administration of low and high doses of a selective noradrenergic reuptake inhibitor did not have a differential effect relative to placebo on performance on the IGT. It is possible that a single dose of a selective noradrenergic reuptake inhibitor was not sufficient to produce a behavioural effect, and that long term administration is required. However, we have shown (using the same protocol) that a single dose of $8 \mathrm{mg}$ reboxetine had a significant effect in impairing long term memory for emotional material. ${ }^{12}$ In addition, Bechara et $a l^{11}$ reported that single doses of both serotonergic and dopaminergic agonists and antagonists had highly significant effects on IGT performance. It could also be argued that we had a comparatively small number of subjects in each group to test our hypothesis, and that our study was under-powered. However, firstly, as mentioned above, we demonstrated a significant behavioural effect (impairment of long term emotional memory ${ }^{12}$ ) with the same sample size and secondly Bechara $e t$ al ${ }^{11}$ reported highly significant effects on IGT performance after drug administration with nine subjects in each of their groups, and finally, our experiment had sufficient power to detect an effect of card deck at $p<0.001$.

Damasio and colleagues have proposed that signals sent via the spinal cord, the vagus, and other cranial nerves, and chemical messages sent via the bloodstream, acting at the level of the brain stem may all be possible routes for "gut feelings" to feedback to the brain and influence higher cognitive functioning. We have previously shown that feedback via the spinal route is unlikely to be involved, as patients with C6 lesions performed the IGT in a manner that was indistinguishable from matched healthy control participants. ${ }^{4}$ Our study also suggests that the noradrenergic system may not be critically involved.

\section{ACKNOWLEDGEMENTS}

We would like to thank Antoine Bechara for providing us with a copy of the Iowa Gambling Task, and Klaus Ebmeier, Polash Shajahan and Mary Stewart for their help in conducting this experiment.

\section{Authors' affiliations}

R O'Carroll, School of Psychology, University of St Andrews, St Andrews, Fife, UK

B Papps, Department of Psychology, Henry Wellcome Building, Institute of Psychiatry, London, UK
Competing interests: none declared.

Correspondence to: Professor R O'Carroll, School of Psychology University of St Andrews, St Andrews, Fife KY16 9JU, UK; e-mail ronan@st-and.ac.uk

Received 13 March 2002

Accepted in revised form 28 November 2002

\section{REFERENCES}

1 Damasio AR. Descartes' error. London: Papermac/Macmillan, 1994.

2 Damasio AR. Towards a neuropathology of emotion and mood. Nature 1997;386:769-827.

3 Damasio A. The feeling of what happens . London: William Heinemann, 1999.

4 North NT, O'Carroll RE Decision making in patients with spinal cord damage: afferent feedback and the somatic marker hypothesis. Neuropsychologia 2001;39:521-4.

5 Bechara A, Damasio AR, Damasio $\mathrm{H}$, et al. Insensitivity to future consequences following damage to human prefrontal cortex. Cognition 1994;50:7-15.

6 Bechara A, Tranel D, Damasio $H$, et al. Failure to respond autonomically to anticipated future outcomes following damage to prefrontal cortex. Cereb Cortex 1996:6:215-25.

7 Bechara A, Damasio H, Tranel D, et al. Deciding advantageously before knowing the advantageous strategy. Science 1997;275:1293-5.

8 Bechara A, Tranel D, Wilson J, et al. Impaired decision-making in peripheral neuropathy. Society for Neuroscience Abstracts 1998:24:1176.

9 Galvez R, Mesches M, McGaugh JL. Norepinephrine release in the amygdala in response to footshock stimulation. Neurobiol Learn Mem 1996:66:253-7

10 Cahill L. Modulation of long-term memory storage in humans by emotional arousal: adrenergic activation and the amygdala. In: Aggleton J, ed. The Amygdala: a functional analysis. Oxford: Oxford University Press, 2000:425-44.

11 Bechara A, Damasio $\mathrm{H}$, et al. Manipulation of dopamine and serotonin causes different effects on covert and overt decision-making. Society for Neuroscience Abstracts 2001;27:126.

12 Papps BP, Shajahan PM, Ebmeier KP, et al. The effects of noradrenergic re-uptake inhibition on memory encoding in man. Psychopharmacology 2002;159:311-18

13 Kerr JS, Powell J, Hindmarch I. The effects of reboxetine and amitriptyline, with and without alcohol on cognitive function and psychomotor performance. Br J Clin Pharmacol 1996;42:239-41.

14 Herman WM, Fuder H. Reboxetine, a selective noradrenaline reuptake inhibitor, is non-sedative and does not impair psychomotor performance in healthy subjects. Hum Psychopharmacol 1998;13:425-33.

15 Dostert P, Benedetti MS, Poggesi I. Review of the pharmacokinetics and metabolism of reboxetine, a selective noradrenaline reuptake inhibitor. Eur Neuropsychopharmacol 1997;7 (suppl 1):S23-35, discussion S71-3.

16 Nelson HE, Willison JR. The revised National Adult Reading Test - Test Manual. Windsor: NFER-Nelson, 1991

17 Eysenck SBG, Eysenck HJ, Barrett P. A revised version of the psychotocism scale. Personality and Individual Differences 1985;6:21-9. 